\title{
A near-infrared view of AGB stars in nearby dwarf galaxies
}

\author{
M. Gullieuszik ${ }^{1}$, E. V. Held ${ }^{1}$, L. Girardi ${ }^{1}$, L. Rizzi $^{2}$, P. Marigo ${ }^{3}$, \\ I. Saviane \& Y. Momany $^{1,4}$ \\ ${ }^{1}$ Osservatorio Astronomico di Padova, INAF, vicolo dell'Osservatorio 5, 35122 Padova, Italy \\ ${ }^{2}$ Joint Astronomy Centre, 660 N. A'ohoku Place, University Park, Hilo, HI 96720, USA \\ ${ }^{3}$ Dip. di Astronomia, Università di Padova, Vicolo dell'Osservatorio 2, 35122 Padova, Italy \\ ${ }^{4}$ European Southern Observatory, Casilla 19001, Santiago 19, Chile
}

\begin{abstract}
As part of our near-infrared photometric survey of nearby dwarf galaxies, we present recent results for Leo I and Leo II dwarf spheroidal galaxies. We selected O- and C-rich AGB stellar populations using two-color diagrams and compared their luminosity functions and star counts with the predictions of the most recent AGB theoretical models.
\end{abstract}

Keywords. stars: AGB and post-AGB, stars: carbon, stars: evolution, galaxies: stellar content,

\section{Observations and data reduction}

We obtained $\mathrm{JHK}_{\mathrm{s}}$-band observations of the Leo I and Leo II Local Group dwarf spheroidal galaxies using the wide-field near-IR camera WFCAM at the UKIRT telescope on Mauna Kea, Hawaii.

The raw data were processed using the WFCAM pipeline provided by the VISTA Data Flow System Project and point spread function photometry was performed on the individual oversampled images ( 6 in $J$ and $H, 10$ in $K_{s}$ ) using the ALLSTAR/ALLFRAME programs. Raw photometric catalogues were calibrated on to the system defined by the 2MASS PSC, by applying the color terms between the WFCAM and 2MASS systems (Dye et al. 2006). The photometric zero-points were then calculated by comparing our transformed magnitudes with the 2MASS magnitudes for stars in common with the PSC.

\section{Selection of AGB stars and comparison with theoretical models}

We used the two-color diagram to select carbon and oxygen-rich stars, and disentangle the foreground Milky Way stellar population. Figure 1 shows our classification for Leo I (Held et al. to be submitted to MNRAS), but the same technique was applied also to Leo II photometry (see Gullieuszik et al. 2008). Using the loci of giant stars and mainsequence dwarf stars defined by Bessell \& Brett (1988), and comparing this diagram with a similar plot obtained for a field beyond the tidal radius of Leo I, we conclude that all stars in regions 2 and 3 are Milky Way dwarfs. Stars in regions 1 and 4 are found only in the field centered on Leo I, and are all Leo I members. Being brighter than the TRGB, they can only be AGB stars. From their position in the CMD (see Fig. 1), we conclude that stars in the regions 1 and 4 are O- and C-rich stars, respectively.

The photometry of Leo I and Leo II was compared with the recent set of TP-AGB evolutionary tracks by Marigo \& Girardi (2007), using the latest version of the TRILEGAL population synthesis code (Girardi \& Marigo 2007) to simulate both the Milky Way foreground and the two galaxies. With respect to the C-rich TP-AGB stars, the comparison between Leo II data and AGB simulations is satisfactory, in terms of location in the 

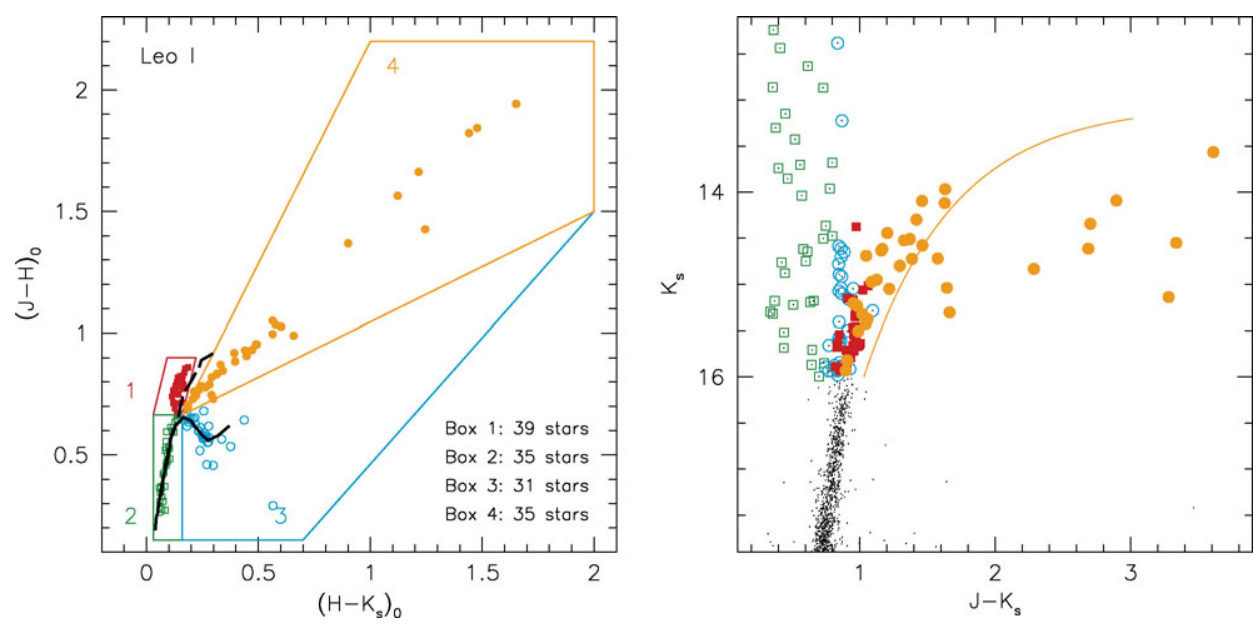

Figure 1. Left Panel: Two-color diagram for stars brighter than the RGB tip in the Leo I field and the loci of giant stars (dashed line) and main-sequence stars (solid line) from Bessell \& Brett (1988). Right Panel: The CMD in the same field. The color-coding represents the selection obtained from the two-color diagram.

CMD, counts and luminosity functions. For O-rich TP-AGB stars, the most important discrepancy consists in an over-estimated prediction of their number and mean $K_{s}$-band luminosities, which could be related to the mass loss prescriptions in our models. Indeed, an increase of mass-loss efficiency, anticipating the superwind phase in O-rich models of low mass and metallicity may constitute an interesting solution to the discrepancy. For Leo I the situation regarding O-rich and C-rich AGB star count is quite different. The number of O-rich stars is correctly predicted but they are $0.5 \mathrm{mag}$ brighter than observed. Simulated C-stars are 2 times more numerous and fainter on the mean than observed, suggesting that our models likely overestimate the lifetime of low- and intermediate-mass C-type stars, and/or underestimate their luminosities. The difference in the two galaxies is likely related to the different mean ages of AGB stars, Leo II stellar populations being significantly older on average than those in Leo I.

To conclude, our work on Leo I and Leo II showed that, despite the possible uncertainties in the star-formation and chemical evolution histories of nearby dwarf galaxies, their number of AGB stars are significant enough to provide valid constraints to AGB stellar models at low metallicities, which are very important since present TP-AGB models highly rely on reproducing the properties of the more metal-rich Magellanic Clouds.

\section{References}

Bessell, M. S. \& Brett, J. M. 1988, PASP, 100, 1134

Dye, S., et al. 2006, MNRAS, 372, 1227

Girardi, L. \& Marigo 2007, in: F. Kerschbaum, C. Charbonnel, \& R. F. Wing (eds), Why Galaxies Care About AGB Stars: Their Importance as Actors and Probes, ASP Conf. Series, Vol. 378, p 20

Gullieuszik, M., Held, E. V., Rizzi, L., Girardi, L., Marigo, P., \& Momany, Y. 2008, MNRAS, 388,1185

Marigo \& Girardi 2007, A\& $A, 469,239$ 